\title{
Reliable reference genes for the quantification of mRNA in human T-cells and PBMCs stimulated with live influenza virus
}

\author{
Justin G. Roy, Janet E. McElhaney and Chris P. Verschoor ${ }^{*}$ (D)
}

\begin{abstract}
Background: Quantitative PCR (qPCR) is a powerful tool that is particularly well-suited to measure mRNA levels in clinical samples, especially those with relatively low cell counts. However, a caveat of this approach is that reliable, stably expressed reference (housekeeping) genes are vital in order to ensure reproducibility and appropriate biological inference. In this study, we evaluated the expression stability of six reference genes in peripheral blood mononuclear cells (PBMCs) and isolated $\mathrm{CD}^{+}$T-cells from young and old adults $(n=10)$, following ex vivo stimulation with mock (unstimulated) or live influenza virus. Our genes included: $\beta$-actin (ACTB), glyercaldehyde-3-phostphate dehydrogenase (GAPDH), ribosomal protein L13a (RPL13a), ribosomal protein S18 (RPS18), succinate dehydrogenase complex flavoprotein subunit A (SDHA), and ubiquitin-conjugating enzyme E2D2 (UBE2D2).
\end{abstract}

Results: Reference gene expression varied significantly depending on cell type and stimulation conditions, but not age. Using the comparative $\Delta C t$ method, and the previously published software BestKeeper, NormFinder, and geNorm, we show that in PBMCs and T-cells, UBE2D2 and RPS18 were the most stable reference genes, followed by ACTB; however, the expression of UBE2D2 and RPS18 was found to increase with viral stimulation in isolated T-cells, while ACTB expression did not change significantly. No age-related differences in stability were observed for any gene

Conclusions: This study suggests the use of a combination of UBE2D2, RPS18, and ACTB for the study of influenza responses in PBMCs and T-cells, although ACTB alone may be the most optimal choice if choosing to compare target gene expression before and after viral stimulation. Both GAPDH and RPL13a were found to be poor reference genes and should be avoided for studies of this nature.

Keywords: Influenza, Human peripheral blood mononuclear cells, T-cells, Reference genes, Housekeeping genes, Quantitative PCR

\section{Background}

A powerful tool one can use to measure gene expression in immune cells, including human peripheral blood mononuclear cells (PBMCs) and T-cells, is quantitative polymerase chain reaction (qPCR). This technology provides high sensitivity when measuring mRNA [1], making it an ideal tool for gene expression analysis in fresh PBMCs and isolated Tcells. An important component of any well-designed qPCR study is the reference or housekeeping gene(s) employed,

\footnotetext{
* Correspondence: cverschoor@hsnri.ca

Health Sciences North Research Institute, 41 Ramsey Lake Rd, Sudbury, ON P3E5J1, Canada
}

which must be chosen carefully and measured precisely $[2$, 3]. These genes are absolutely critical in order to control for differences in overall transcript abundance from sample to sample, and as such, have substantial influence on measured levels of target mRNAs $[4,5]$. Therefore, before attempting to evaluate changes in gene expression within human PBMC and T-cell populations, the identification of stable reference genes must be performed under the conditions which they will be measured. Unfortunately, it has been demonstrated that no single gene can be used for all cell types and tissue types [6], and in some cases, multiple reference genes are required [7]. 
This study aimed to identify stable reference genes in human PBMCs and $\mathrm{CD}^{+} \mathrm{T}$-cells for an assay commonly used to measure immunity following infection or vaccination to influenza A virus, a major respiratory pathogen $[8,9]$. In addition to comparing reference gene stability with and without live influenza challenge ex vivo, we also compared stability between young and old adults, given that age is a major determinant of susceptibility to infection and can alter PBMC mRNA expression profiles significantly [10, 11]. Six candidate reference genes, chosen from the literature, were assessed in human PBMCs and Tcells. Commonly used genes such as $A C T B$ and GAPDH were included even though many studies have demonstrated high variability in the expression of these genes under various conditions [2, 4, 12-14], including influenza infection [15]. RPL13a and SDHA have been found to be valid reference genes in both T-cells and mixed leukocytes [7], while UBE2D2 was found to be the most stably expressed reference gene in different PBMC subsets of Multiple Sclerosis patients [16]. Finally, RPS18 was included as it has been shown to be fairly stable in PBMCs from other species [17] and in tumour neovascularization studies [18]. We compared these genes using four methods, each of which estimating stability and/or reliability in a slightly differ manner: geNorm [7] determines gene expression stability (ie. M) by calculating the average pairwise variation of each reference gene; NormFinder [19] uses an ANOVA based approached to calculate the candidate gene stability value by estimating the expression variation within the overall group (intragroup) and between groups (intergroup); Bestkeeper [20] estimates reliability according to the standard deviation of $\mathrm{Cq}$ values and the Pearson correlation between a given gene and an index of the most stable reference genes, as determined by the software; Lastly, the comparative $\Delta \mathrm{Ct}$ method, proposed by Silver and colleagues [21], compares the relative expression of pairs of reference genes within the sample and uses the average standard deviation of the $\Delta \mathrm{Ct}$ (or $\Delta \mathrm{Cq}$ ) for each reference gene as a measure of stability.

\section{Results}

Evaluation of candidate reference gene expression in unstimulated and influenza a stimulated PBMCs and Tcells

Using $\mathrm{qPCR}$, the expression of each of the six candidate reference genes (Tables 1 and 2) was measured in PBMCs and T-cells from a combination of young and old donors $(n=$ 10 ), in the presence or absence of influenza stimulation. When results from both treatments and cell types were combined, RPS18 (mean Cq $\sim 20$ ) demonstrated the highest expression, followed by ACTB ( 21.5), GAPDH ( 23.5), UBE2D2 ( 25), SDHA ( 25.5) and RPL13a ( 30.5); no significant difference between cell types were observed. In PBMC samples, both GAPDH $(p<0.001)$ and RPL13a $(p<$ $0.001)$ were significantly different between unstimulated and stimulated treatments; the remaining candidate reference genes showed no significant differences (Fig. 1A). In isolated T-cells, four of six candidate genes were significantly different between treatments: GAPDH $(p<0.01)$, RPL13a $(p<0.01)$, RPS18 $(p<0.001)$, and UBE2D2 $(p<$ 0.001) (Fig. 1B). Age did not appear to have a significant effect on the expression of the candidate reference genes (Additional file 1: Fig. S1).

\section{Analysis of candidate reference genes using Silver's method, geNorm, BestKeeper and NormFinder}

Candidate reference gene expression was further analyzed through the use of previously published software and techniques (Table 3). With exception to the NormFinder approach, all analyses were performed on data pooled from young and old adults, and with and without virus stimulation.

With respect to Silver's method, $U B E 2 D 2$ was determined to have the greatest stability in PBMCs (0.493), followed by ACTB (0.520), RPS18 (0.538) and SDHA (0.564); this was similarly observed in isolated T-cells. For both PBMCs and T-cells, GAPDH was ranked as the least stable gene (1.204 and 1.290, respectively).

According to geNorm, all reference genes considered were deemed stable in both PBMCs and T-cells $(\mathrm{M}<$ 1.5, according to [7]); for both cell types, RPS18 $($ PBMCs $=0.52$, T-cells $=0.278)$, SDHA $(0.53,0.311)$ and UBE2D2 (0.537, 0.287) were ranked the highest.

Table 1 Candidate reference genes selected for this study

\begin{tabular}{lllll}
\hline Symbol & Name & Accession Number & Amplicon Size (bp) & IDT Assay ID \\
\hline ACTB & B-actin & NM_001101.5 & 110 & Hs.PT.39a.22214847 \\
GAPDH & Glyercaldehyde-3-phostphate dehydrogenase & NM_002046.7 & 143 & Hs.PT.39a.22214836 \\
RPL13a & Ribosomal protein, L13a & NM_012423.4 & 106 & Hs.PT.58.45725862 \\
RPS18 & Ribosomal protein, S18 & NM_022551.3 & 130 & Hs.PT.58.14390640 \\
SDHA & Succinate dehydrogenase complex flavoprotein subunit A & NM_004168.4 & 150 & Hs.PT.58.41017719 \\
UBE2D2 & Ubiquitin-conjugating enzyme E2D2 & NM_181838.1 & 119 & Hs.PT.58.622887 \\
\hline
\end{tabular}


Table 2 Candidate reference gene primers and details

\begin{tabular}{|c|c|c|c|}
\hline Symbol & Primer and Probe Sequences $\left(5^{\prime}-3^{\prime}\right)$ & Exons & Average Efficiency (\%) $^{\mathrm{a}}$ \\
\hline$\overline{A C T B}$ & $\begin{array}{l}\text { F: ACAGAGCCTCGCCTITG } \\
\text { R: CCTTGCACATGCCGGAG } \\
\text { P: /5HEX/TCATCCATG/ZEN/GTGAGCTGGCGG/3IABkFQ/ }\end{array}$ & $1-2$ & 99.5 \\
\hline GAPDH & $\begin{array}{l}\text { F: ACATCGCTCAGACACCATG } \\
\text { R: TGTAGTTGAGGTCAATGAAGGG } \\
\text { P: /5HEX/AAGGTCGGA/ZEN/GTCAACGGATTTGGTC/3AIBkFQ/ }\end{array}$ & $2-3$ & 96.8 \\
\hline RPL13a & $\begin{array}{l}\text { F: GCCGCCCCTGTTTCAAG } \\
\text { R: CTCGACCATCAAGCACCAG } \\
\text { P: /5HEX/AGAAACCCT/ZEN/GCGACAAAACCTCCT/3IABkFQ/ }\end{array}$ & $1 b-3$ & 99.3 \\
\hline RPS18 & $\begin{array}{l}\text { F: GTTCCAGCATATITTGCGAGT } \\
\text { R: GTCAATGTCTGCTITCCTCAAC } \\
\text { P: /5HEX/TCTTCGGCC/ZEN/CACACCCTTAATGG/3IABkFQ/ }\end{array}$ & $2-3$ & 99.5 \\
\hline SDHA & $\begin{array}{l}\text { F: TTGGATGCAGTGGTGGTAGG } \\
\text { R: CAGAGCAGCATTGATTCCTC } \\
\text { P: /5HEX/TGCAACAGT/ZEN/GTGTGACCTGGTAGG/3IABkFQ/ }\end{array}$ & $3-4$ & 99.5 \\
\hline UBE2D2 & $\begin{array}{l}\text { F: GTACTCTTGTCCATCTGTTCTCTG } \\
\text { R: CCATTCCCGAGCTATTCTGTT } \\
\text { P: /5HEX/CCGAGCAAT/ZEN/CTCAGGCACTAAAGGA/3IABkFQ/ }\end{array}$ & $6-8$ & 99.3 \\
\hline
\end{tabular}

${ }^{a}$ Average efficiencies were calculated from both unstimulated PBMC and T-cell samples

Interestingly, $A C T B$, which was deemed suitable using Silver's method in PBMCs, had the lowest expression stability value of the reference genes tested (1.28). In Tcells, GAPDH was deemed to be the least stable, however, still within the stability cut-off (0.854).

Using BestKeeper, all reference genes were determined to be relatively stable in both PBMCs and T-cells $(\mathrm{SD}<1)$, according to [20]). In PBMCs, UBE2D2 and RPS18 were the most stable (ie. lowest SD), while exhibiting the highest correlations (UBE2D2: $\mathrm{SD}=0.22, r=0.836 ; R P S 18: \mathrm{SD}=0.25$, $r=0.738$ ), followed by $A C T B(\mathrm{SD}=0.29, r=0.737)$; $S D H A$ exhibited good stability $(\mathrm{SD}=0.26)$, but a relatively low correlation $(r=0.401)$. In T-cells, RPS18 and UBE2D2 demonstrated the best stability and correlation (RPS18: $\mathrm{SD}=0.33$, $r=0.873$; UBE2D2: $\mathrm{SD}=0.35, r=0.907)$, while $A C T B$ and $S D H A$ only exhibited good stability $(0.20$ and 0.29 ,
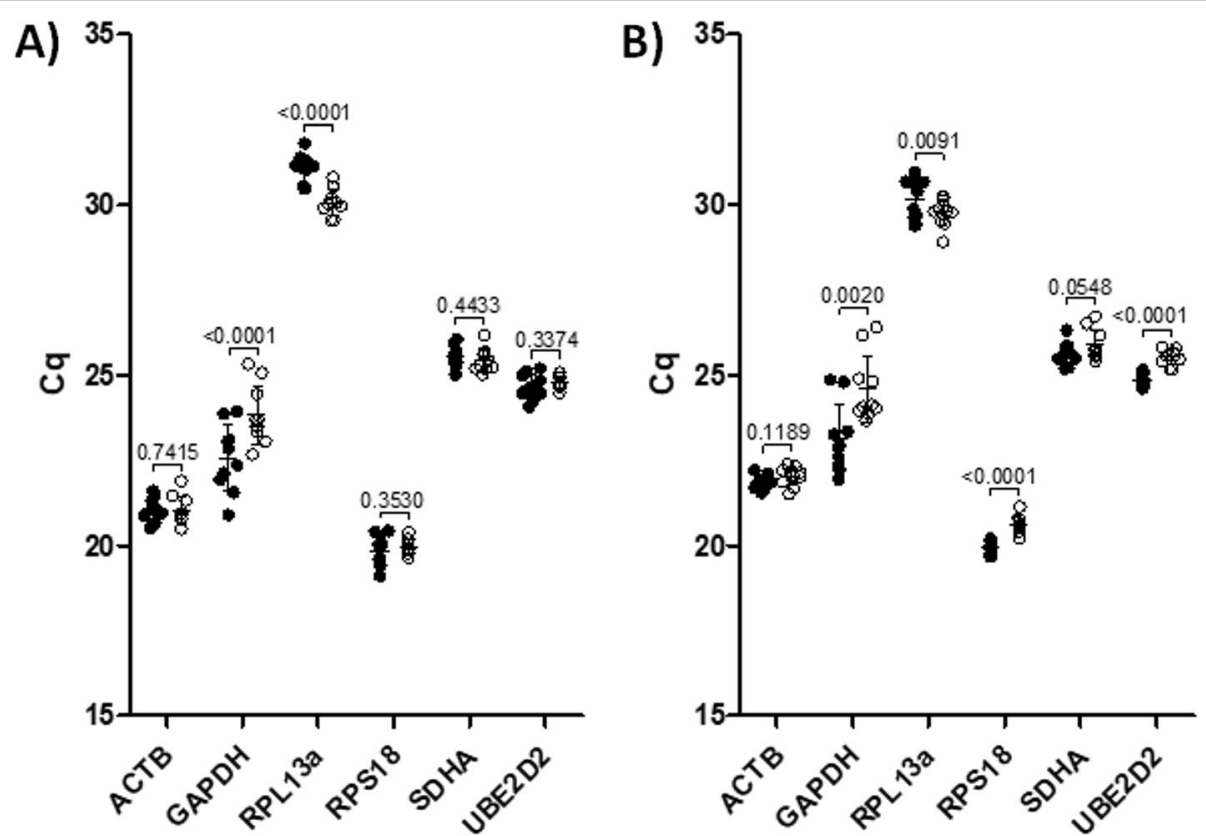

Fig. $1 \mathrm{Cq}$ values for candidate reference genes in stimulated and unstimulated PBMCs and T-cells. Mean quantification cycle (Cq) values are presented for both unstimulated and influenza ANictoria/375 stimulated donor $(n=10)$ PBMCs $(\mathbf{a})$ and $\mathrm{CD}^{+}{ }^{+}$T-cells $(\mathbf{b})$. Unstimulated controls are presented by the black, filled circles, and the paired stimulated samples are presented by clear circles. Statistical analysis was performed by paired t-test or Wilcoxon signed rank test. $P$-values are presented between indicated comparisons. Data from 10 young and old donors is presented 
Table 3 Reference gene reliability analysis on unstimulated and live influenza A virus stimulated PBMCs using Silver's method, geNorm, BestKeeper and NormFinder

\begin{tabular}{|c|c|c|c|c|c|c|c|c|c|c|c|c|c|}
\hline \multirow[b]{2}{*}{ Method } & \multirow[b]{2}{*}{ Measure } & \multicolumn{2}{|l|}{ ACTB } & \multicolumn{2}{|c|}{ GAPDH } & \multicolumn{2}{|l|}{ RPL13a } & \multicolumn{2}{|l|}{ RPS18 } & \multicolumn{2}{|l|}{ SDHA } & \multicolumn{2}{|c|}{ UBE2D2 } \\
\hline & & $\overline{P B M C}$ & $T$-cell & PBMC & $\overline{T \text {-cell }}$ & $\overline{P B M C}$ & T-cell & $\overline{P B M C}$ & T-cell & $\overline{P B M C}$ & T-cell & $\overline{P B M C}$ & T-cell \\
\hline Silver & $\sigma C q$ & 0.52 & 0.562 & 1.204 & 1.29 & 0.803 & 0.821 & 0.538 & 0.568 & 0.564 & 0.601 & 0.493 & 0.541 \\
\hline geNorm & M & 1.281 & 0.362 & 0.825 & 0.854 & 0.556 & 0.312 & 0.52 & 0.278 & 0.53 & 0.311 & 0.537 & 0.287 \\
\hline \multirow[t]{2}{*}{ BestKeeper } & SD & 0.29 & 0.20 & 0.84 & 0.96 & 0.52 & 0.43 & 0.25 & 0.33 & 0.26 & 0.29 & 0.22 & 0.35 \\
\hline & r & 0.737 & 0.504 & 0.587 & 0.688 & 0.227 & -0.173 & 0.738 & 0.873 & 0.401 & 0.468 & 0.836 & 0.907 \\
\hline \multirow[t]{3}{*}{ NormFinder (Stability Value) } & Intragroup (all samples) & 0.067 & 0.100 & 0.810 & 0.873 & 0.479 & 0.496 & 0.068 & 0.074 & 0.192 & 0.219 & 0.073 & 0.074 \\
\hline & Intergroup ( \pm virus) & 0.094 & 0.147 & 0.572 & 0.508 & 0.436 & 0.402 & 0.074 & 0.08 & 0.152 & 0.173 & 0.046 & 0.099 \\
\hline & Intergroup $(\mathrm{Y} / \mathrm{O})$ & 0.034 & 0.039 & 0.26 & 0.281 & 0.154 & 0.161 & 0.044 & 0.029 & 0.058 & 0.066 & 0.022 & 0.023 \\
\hline
\end{tabular}

Expression stability of candidate reference genes was calculated from $n=10$ paired unstimulated and influenza A/Victoria/375 stimulated PBMCs and T-cells

respectively). GAPDH exhibited the worst stability across cell types (PBMCs $=0.84$, T-cells $=0.96$ ), while $R P L 13 a$ exhibited the worst correlation (PBMCs $=$ 0.227, T-cells $=-0.173$ ).

We performed an intragroup analysis of all unstimulated and stimulated samples using NormFinder and found that RPS18, UBE2D2 and $A C T B$ were determined to have the lowest stability value (ie. best stability) in PBMCs and T-cells (Stability Value $\leq 0.10$ ); this was similarly observed for the intergroup analyses, where stratification by treatment or age was considered. Although not deemed as stable as RPS18, UBE2D2 and $A C T B$, the Stability Values observed for $S D H A$ are notable $(\leq 0.219)$.

\section{Scoring the best and worst reference genes according to stability}

For our assessment of reference gene stability, we considered four different approaches and seven measures in total. To summarize these findings and conclude on the best and worst genes evaluated, we ranked each gene and assigned a score of 3, 2 or 1 if the gene was found to rank 1st, 2nd or 3rd best, respectively, for a given measure. For PBMCs, UBE2D2 (score = 17) was best, followed by RPS18 (13), $A C T B$ (9), SDHA (3), and GAPDH/RPL13a (0). For T-cells, $U B E 2 D 2$ and RPS18 scored similarly (15), followed by $A C T B$ (8), SDHA (3), GAPDH (1) and RPL13a (0).

\section{Discussion}

In this study, we evaluated the expression stability and suitability of candidate reference genes in influenza virus stimulated PBMCs and T-cells. Our data shows that $U B E 2 D 2$ and RPS18 ranked the highest with regards to stability in both PBMCs and T-cells, followed closely by $A C T B$. However, both UBE2D2 and RPS18 were expressed significantly higher following viral stimulation in T-cells, but not $A C T B$. Whether considering overall stability or comparing expression with or without viral stimulation, GAPDH and RPL13a were ranked the worst in both cell types.
The software geNorm, NormFinder and Bestkeeper, and Silver's method all provided similar results for both the PBMC and T-cell reference gene analysis, ranking $U B E 2 D 2$ as the most stable gene, followed by RPS18; this has been previously demonstrated $[16,18]$. Furthermore, in PBMCs these genes were found to not be affected by age or viral stimulation. This is particularly important in the context of influenza, given the prominent role of age-related immune dysfunction in determining the susceptibility to infection or response to vaccination [22, 23]. Interestingly, the next highest ranked gene we identified, $A C T B$, has previously been demonstrated to have high variance in expression [14], including in T-cells [3, 24]. While this should suggest caution prior to implementing $A C T B$ as a reference gene, it is worth noting that it was also one of the few genes that was not significantly different in virus stimulated and unstimulated cultures in PBMCs or T-cells. Hence, $A C T B$ may warrant consideration for mRNA studies that are specifically comparing target gene expression before and after immune stimulation, especially since both $U B E 2 D 2$ and RPS18 were significantly different with viral stimulation in isolated T-cells. As a whole, this demonstrates the importance of testing each specific experimental condition on potential reference genes as well as in specific sample types, prior to the measurement of target genes.

Our study has a number of strengths resulting in a comprehensive analysis that will help researchers decide on the best reference gene to use depending on their experimental conditions and study design. We compared six well-known reference genes using four different approaches and seven measures in total, and also stratified our analysis by cell type (PBMCs and isolated T-cells), age group (young and old), and experimental treatment (with and without virus). However, we concede that experimental conditions beyond that tested in our study may impact the stability of reference genes differently. Furthermore, it is possible that we were underpowered to investigate the effect of age on reference gene 
stability, given that others have shown there to be a notable effect, albeit in tissues [25, 26].

\section{Conclusions}

The current Minimum Information for Publication of Quantitative Real-Time PCR Experiments (MIQE) suggests the use of more than one reference genes in all qPCR studies [4]. Hence, we recommend the use of UBE2D2 and RPS18, or UBE2D2, RPS18 and ACTB in studies of PBMCs or isolated T-cells. However, when comparing target gene expression across stimulations, we recommend the use of $A C T B$ alone if studying isolated T-cells. In our analyses neither GAPDH or RPL13a were found to be reliable reference genes, and should be avoided unless properly evaluated under the intended research conditions.

\section{Methods}

\section{Study design and participants}

Frozen PBMC samples from a previous study [27] were used for the identification of reference genes. Ten samples, derived from heparinized blood collected 4-weeks following influenza vaccination, were chosen and consisted of five older donors $(\mathrm{O}$; mean age [range] $=77$ [70-80]) and five younger donors (Y; 30 [26-35]), and similarly balanced according to cytomegalovirus serostatus $(\mathrm{O}=40 \%$ seropositive, $\mathrm{Y}=60 \%$ seropositive $)$.

\section{PBMC cell culture, stimulation and $\mathrm{CD}^{+}{ }^{+} \mathrm{T}$-cell isolation}

To measure candidate reference gene expression, PBMCs were stimulated with live influenza virus, as previously described with some changes [28]. Briefly, frozen PBMC samples [27] were thawed, counted and adjusted to a concentration of $1 \times 10^{6}$ cells $/ 200 \mu \mathrm{L}$ in Aim V media (Gibco) supplemented with Human AB Serum (Corning). PBMCs were placed in 96-well U-bottom plates (Corning) and were either left unstimulated or stimulated with live influenza A/Victoria/375 virus (sucrose-gradient purified, Charles River Laboratories) at a multiplicity of infection of six for $8 \mathrm{~h}$ at $37^{\circ} \mathrm{C}, 5 \% \mathrm{CO}_{2}$ in a humidified chamber. After incubation, cells for PBMC mRNA analysis were collected and stored in $350 \mu \mathrm{L}$ RLT buffer (Qiagen) supplemented with $1 \% \beta$-mercaptomethanol (Sigma-Aldrich) at $-80^{\circ} \mathrm{C} . \mathrm{CD}^{+} \mathrm{T}$-cells were isolated from remaining cells using the EasySep Human T-cell Enrichment kit (Stem Cell Technologies), following manufacturer's instructions and stored in RLT buffer at $-80^{\circ} \mathrm{C}$.

\section{RNA isolation and CDNA synthesis}

RNA was isolated from PBMC and T-cell lysates using the RNeasy Mini Kit (Qiagen) according to manufacturer's instructions. Isolated RNA was quantified using the NanoDrop 2000 Spectrophotometer (Thermo Scientific) and stored at $-80^{\circ} \mathrm{C}$; RNA with an $260 / 280$ absorbance ratio below 1.75 were not tested further.
Complementary DNA (cDNA) was synthesized from $100 \mathrm{ng}$ of total RNA using of the High Capacity cDNA reverse transcription kit (Applied Biosystems) according to manufacturer's recommendation (final volume $=$ $20 \mu \mathrm{L}$ ), and stored at $-20^{\circ} \mathrm{C}$.

\section{Quantitative PCR and reference genes}

Candidate reference gene primer/probe mixes were purchased from Integrated DNA Technologies as prevalidated probe PrimeTime ${ }^{\circ}$ PCR Probe Assays using TaqMan based chemistry (Table 1). Table 2 highlights the primer and probe sequences, location of the primers and the average efficiencies of the primers in previous experiments using 2-fold serial dilutions; primer efficiency was calculated as $10^{(-1 / \text { slope })}$. Quantitative PCR was performed using the QuantStudio ${ }^{\mathrm{m}} 5$ Real Time PCR System (Applied Biosystems); briefly, $2 \mu \mathrm{L}$ of cDNA of each sample was loaded in duplicate into 96-well plates and $8 \mu \mathrm{L}$ of qPCR master mix was added, which included TaqMan Fast Advanced Master Mix $(5 \mu \mathrm{L}$; Applied Biosystems), reference gene primer/probe mix $(0.5 \mu \mathrm{L})$, and RNase/DNase free $\mathrm{H}_{2} \mathrm{O}(2.5 \mu \mathrm{L}$; Qiagen)). Negative controls (no RNA or cDNA) were included to verify the absence of contamination. Amplification was performed at $60^{\circ} \mathrm{C}$ using a two-step cycling procedure for 40 cycles, and resultant quantification cycles (Cq) were calculated using the default settings in the QuantStudio Design \& Analysis Software v1.4.3 (Applied Biosystems).

\section{Statistical analysis and identification of suitable reference genes}

All statistical tests were performed using GraphPad Prism 5. Pairwise comparisons were performed by either the Wilcoxon signed ranked sum test or a Student's paired ttest, depending on the normality of data, which was determined by the Shapiro-Wilks normality test. To evaluate reference gene stability, four separate methods were employed: geNorm [7], NormFinder [19], BestKeeper [20], and Silver's $\Delta \mathrm{Ct}$ method [21]. For geNorm and NormFinder analyses, mean $\mathrm{Cq}$ values were transformed into a linear scale $\left(2^{-\Delta C q}\right.$, where $\Delta C q=C q-$ minimum $\left.C q\right)$; for both BestKeeper and Silver's method, mean $\mathrm{Cq}$ values were used. From these analyses we obtained the following measures: $\sigma_{C q}$ (Silver's method), which represents the average standard deviation of the difference in cycle threshold values across all possible pairs of reference genes; $\mathrm{M}$ (geNorm), a measure of gene expression stability relative to all candidate reference genes; SD (BestKeeper), the raw standard deviation of $\mathrm{Cq}$ values relative to the software's index measure; $r$ (BestKeeper), the coefficient of correlation relative to the software's index measure; and the Stability Value (NormFinder), representing expression variance in both the intragroup and intergroup analyses. 


\section{Supplementary information}

Supplementary information accompanies this paper at https://doi.org/10. 1186/s12865-020-0334-8

Additional file 1: Figure S1. Cq values for candidate reference genes in PBMCs and T-cells, stratified by age and stimulation status. Mean quantification cycle $(\mathrm{Cq})$ values are presented for young $(\mathrm{YN})$ and old $(\mathrm{ON})$ unstimulated ( - ) and influenza ANictoria/375 stimulated (+) donor PBMCs $(A)$ and CD3+ T-cells (B). No significant differences $(p>0.20)$ were detected by Wilcoxon rank-sum test and data from a total of 10 donors is presented.

\section{Abbreviations}

ACTB: $\beta$-actin; $\mathrm{CD}^{+}$: Cluster of differentiation 3; cDNA: Complementary deoxyribonucleic acid; CMV: Cytomegalovirus; Cq: Cycle of quantification; GAPDH: Glyercaldehyde-3-phostphate dehydrogenase; MIQE: The Minimum Information for Publication of Quantitative Real-Time PCR Experiments; O: Old participant: PBMCs: Peripheral blood mononuclear cells; PGK1: Phosphoglycerate kinase 1; qPCR: Quantitative polymerase chain reaction; rcf: Relative centrifugal force; RNA: Ribonucleic acid; RPL13a: Ribosomal protein, L13a; RPLP0: Ribosomal protein large, P0; RPS18: Ribosomal protein, S18; SD: Standard deviation; SDHA: Succinate dehydrogenase complex flavoprotein subunit A; UBE2D2: Ubiquitinconjugating enzyme E2D2; Y: Young participant; $\sigma_{\mathrm{Cq}}$ : Average standard deviation of $\Delta \mathrm{Ct}$ values across all possible pairs of reference genes

\section{Acknowledgements}

The authors would like to thank Beth Gentleman for the aid provided in preparing the Research Ethics Board application.

\section{Authors' contributions}

JGR designed and performed the experiments, analyzed the results, and drafted the manuscript. JGR, JEM and CPV edited the draft for final submission. All authors read and approved the final manuscript.

\section{Funding}

This study was supported by the Academic Health Science Centre Alternative Funding Plan Innovation Fund, Project Code A-13-04 and A-17-07 procured by JEM.

\section{Availability of data and materials}

The datasets used and/or analysed during the current study are available from the corresponding author on reasonable request.

\section{Ethics approval and consent to participate}

Written informed consent was obtained for all participants included in this study. The Health Sciences North Research Institute Research Ethics Board approved the study (\#19-026).

\section{Consent for publication}

Not applicable.

\section{Competing interests}

The authors declare that they have no competing interests.

Received: 20 September 2019 Accepted: 20 January 2020 Published online: 31 January 2020

\section{References}

1. Bustin SA, Benes V, Nolan T, Pfaffl MW. Quantitative real-time RT-PCR--a perspective. J Mol Endocrinol. 2005;34:597-601.

2. Dheda K, Huggett JF, Chang JS, Kim LU, Bustin SA, Johnson MA, et al. The implications of using an inappropriate reference gene for real-time reverse transcription PCR data normalization. Anal Biochem. 2005;344:141-3.

3. Thellin O, EIMoualij B, Heinen E, Zorzi W. A decade of improvements in quantification of gene expression and internal standard selection. Biotechnol Adv. 2009;27:323-33.

4. Bustin SA, Benes V, Garson JA, Hellemans J, Huggett J, Kubista M, et al. The MIQE guidelines: minimum information for publication of quantitative realtime PCR experiments. Clin Chem. 2009;55:611-22.
5. Jacob F, Guertler R, Naim S, Nixdorf S, Fedier A, Hacker NF, et al. Careful selection of reference genes is required for reliable performance of RT-qPCR in human normal and cancer cell lines. PLoS One. 2013;8:e59180.

6. Huggett J, Dheda K, Bustin S, Zumla A. Real-time RT-PCR normalisation; strategies and considerations. Genes Immun. 2005;6:279-84.

7. Vandesompele J, De Preter K, Pattyn F, Poppe B, Van Roy N, De Paepe A, et al. Accurate normalization of real-time quantitative RT-PCR data by geometric averaging of multiple internal control genes. Genome Biol. 2002; 3. https://doi.org/10.1186/gb-2002-3-7-research0034.

8. McElhaney JE, Gentleman B. Cell-mediated immune response to influenza using ex vivo stimulation and assays of cytokine and Granzyme B responses. Methods Mol Biol. 2015;1343:121-41.

9. Haq K, Fulop T, Tedder G, Gentleman B, Garneau H, Meneilly GS, et al. Cytomegalovirus Seropositivity predicts a decline in the T cell but not the antibody response to influenza in vaccinated older adults independent of type 2 diabetes status. J Gerontol Biol Sci Med Sci. 2017;72:1163-70.

10. Zhou X, McElhaney JE. Age-related changes in memory and effector T cells responding to influenza a/H3N2 and pandemic a/H1N1 strains in humans. Vaccine. 2011:29:2169-77.

11. Steegenga WT, Boekschoten MV, Lute C, Hooiveld GJ, de Groot PJ, Morris $\mathrm{TJ}$, et al. Genome-wide age-related changes in DNA methylation and gene expression in human PBMCs. AGE. 2014;36:9648.

12. Bas A, Forsberg G, Hammarstrom S, Hammarstrom M-L. Utility of the housekeeping genes $18 \mathrm{~S}$ rRNA, beta-actin and Glyceraldehyde-3-phosphatedehydrogenase for normalization in real-time quantitative reverse transcriptase-polymerase chain reaction analysis of gene expression in human T Iymphocytes. Scand J Immunol. 2004;59:566-73.

13. Piehler AP, Grimholt RM, Øvstebø R, Berg JP. Gene expression results in lipopolysaccharide-stimulated monocytes depend significantly on the choice of reference genes. BMC Immunol. 2010;11:21.

14. Chapman JR, Waldenström J. With reference to reference genes: a systematic review of endogenous controls in gene expression studies. PLoS One. 2015;10. https://doi.org/10.1371/journal.pone.0141853.

15. Kuchipudi SV, Tellabati M, Nelli RK, White GA, Perez BB, Sebastian S, et al. $18 \mathrm{~S}$ rRNAis a reliable normalisation gene for real time PCR based on influenza virus infected cells. Virol J. 2012;9. https://doi.org/10.1186/1743$422 X-9-230$

16. Oturai DB, Søndergaard HB, Börnsen L, Sellebjerg F, Romme CJ. Identification of suitable reference genes for peripheral blood mononuclear cell subset studies in multiple sclerosis. Scand J Immunol. 2016;83:72-80.

17. Chen I-H, Chou L-S, Chou S-J, Wang J-H, Stott J, Blanchard M, et al. Selection of suitable reference genes for normalization of quantitative RTPCR in peripheral blood samples of bottlenose dolphins (Tursiops truncatus) Sci Rep. 2015;5. https://doi.org/10.1038/srep15425.

18. Rienzo M, Schiano C, Casamassimi A, Grimaldi V, Infante T, Napoli C. Identification of valid reference housekeeping genes for gene expression analysis in tumor neovascularization studies. Clin Transl Oncol. 2013;15:211-8

19. Andersen $\mathrm{CL}$, Jensen JL, Ørntoft TF. Normalization of real-time quantitative reverse transcription-PCR data: a model-based variance estimation approach to identify genes suited for normalization, applied to bladder and Colon Cancer data sets. Cancer Res. 2004;64:5245-50.

20. Pfaffl MW, Tichopad A, Prgomet C, Neuvians TP. Determination of stable housekeeping genes, differentially regulated target genes and sample integrity: BestKeeper - excel-based tool using pair-wise correlations. Biotechnol Lett. 2004;26:509-15.

21. Silver N, Best S, Jiang J, Thein S. Selection of housekeeping genes for gene expression studies in human reticulocytes using real-time PCR. BMC Mol Biol. 2006;7. https://doi.org/10.1186/1471-2199-7-33.

22. Falsey AR, Hennessey PA, Formica MA, Cox C, Walsh EE. Respiratory syncytial virus infection in elderly and high-risk adults. N Engl J Med. 2005:352:1749-59.

23. McElhaney JE, Xie D, Hager WD, Barry MB, Wang Y, Kleppinger A, et al. T cell responses are better correlates of vaccine protection in the elderly. J Immunol Baltim Md 1950. 2006;176:6333-9.

24. Ledderose C, Heyn J, Limbeck E, Kreth S. Selection of reliable reference genes for quantitative real-time PCR in human T cells and neutrophils. BMC Res Notes. 2011;4. https://doi.org/10.1186/1756-0500-4-427.

25. Harrison OJ, Moorjani N, Torrens C, Ohri SK, Cagampang FR. Endogenous reference genes for gene expression studies on bicuspid aortic valve associated Aortopathy in humans. PLoS One. 2016;11:e0164329. 
26. Timaru-Kast R, Herbig EL, Luh C, Engelhard K, Thal SC. Influence of age on cerebral housekeeping gene expression for normalization of quantitative polymerase chain reaction after acute brain injury in mice. J Neurotrauma. 2015:32:1777-88.

27. Wagar LE, Gentleman B, Pircher H, McElhaney JE, Watts TH. Influenzaspecific $T$ cells from older people are enriched in the late effector subset and their presence inversely correlates with vaccine response. PLoS One. 2011;6. https://doi.org/10.1371/journal.pone.0023698.

28. McElhaney JE, Gentleman B. Cell-mediated immune response to influenza using ex vivo stimulation and assays of cytokine and Granzyme B responses. In: Shaw AC, editor. Immunosenescence: methods and protocols. New York: Springer New York; 2015. p. 121-41. https://doi.org/10.1007/978-1-4939-2963-4_11.

\section{Publisher's Note}

Springer Nature remains neutral with regard to jurisdictional claims in published maps and institutional affiliations.

Ready to submit your research? Choose BMC and benefit from:

- fast, convenient online submission

- thorough peer review by experienced researchers in your field

- rapid publication on acceptance

- support for research data, including large and complex data types

- gold Open Access which fosters wider collaboration and increased citations

- maximum visibility for your research: over $100 \mathrm{M}$ website views per year

At $\mathrm{BMC}$, research is always in progress.

Learn more biomedcentral.com/submissions 